\title{
Serum progesterone distribution in normal pregnancies compared to pregnancies complicated by threatened miscarriage from 5 to 13 weeks gestation: a prospective cohort study
}

\author{
Chee Wai Ku ${ }^{*}$, John C. Allen $\mathrm{Jr}^{2}$, Sze Min Lek ${ }^{1}$, Ming Li Chia ${ }^{3}$, Nguan Soon Tan ${ }^{4,5}$ and Thiam Chye Tan ${ }^{1,2}$
}

\begin{abstract}
Background: Progesterone is a critical hormone in early pregnancy. A low level of serum progesterone is associated with threatened miscarriage. We aim to establish the distribution of maternal serum progesterone in normal pregnancies compared to pregnancies complicated by threatened miscarriage from 5 to 13 weeks gestation.

Methods: This is a single centre, prospective cohort study of 929 patients. Women from the Normal Pregnancy [NP] cohort were recruited from antenatal clinics, and those in the Threatened Miscarriage [TM] cohort were recruited from emergency walk-in clinics. Women with multiple gestations, missed, incomplete or inevitable miscarriage were excluded from the study. Quantile regression was used to characterize serum progesterone levels in the NP and TM cohorts by estimating the 10th, 50th and 90th percentiles from 5 to 13 weeks gestation. Pregnancy outcome was determined at 16 weeks of gestation. Subgroup analysis within the TM group compared progesterone levels of women who subsequently miscarried with those who had ongoing pregnancies at 16 weeks of gestation.

Results: Median serum progesterone concentration demonstrated a linearly increasing trend from $57.5 \mathrm{nmol} / \mathrm{L}$ to $80.8 \mathrm{nmol} / \mathrm{L}$ from 5 to 13 weeks gestation in the NP cohort. In the TM cohort, median serum progesterone concentration increased from $41.7 \mathrm{nmol} / \mathrm{L}$ to $78.1 \mathrm{nmol} / \mathrm{L}$. However, median progesterone levels were uniformly lower in the TM cohort by approximately $10 \mathrm{nmol} / \mathrm{L}$ at every gestation week. In the subgroup analysis, median serum progesterone concentration in women with ongoing pregnancy at 16 weeks gestation demonstrated a linearly increasing trend from 5 to 13 weeks gestation. There was a marginal and non-significant increase in serum progesterone from 19.0 to $30.3 \mathrm{nmol} / \mathrm{L}$ from 5 to 13 weeks gestation in women who eventually had a spontaneous miscarriage.

Conclusions: Serum progesterone concentration increased linearly with gestational age from 5 to 13 weeks in women with normal pregnancies. Women with spontaneous miscarriage showed a marginal and non-significant increase in serum progesterone. This study highlights the pivotal role of progesterone in supporting an early pregnancy, with lower serum progesterone associated with threatened miscarriage and a subsequent complete miscarriage at 16 weeks gestation.
\end{abstract}

Keywords: Serum progesterone, First trimester distribution, Progesterone nomogram, Threatened miscarriage

\footnotetext{
* Correspondence: cheewai.ku@mohh.com.sg

'Department of Obstetrics and Gynecology, KK Women's and Children's

Hospital, Singapore, 100 Bukit Timah Road, Singapore 229899, Singapore

Full list of author information is available at the end of the article
}

(c) The Author(s). 2018 Open Access This article is distributed under the terms of the Creative Commons Attribution 4.0 International License (http://creativecommons.org/licenses/by/4.0/), which permits unrestricted use, distribution, and

reproduction in any medium, provided you give appropriate credit to the original author(s) and the source, provide a link to the Creative Commons license, and indicate if changes were made. The Creative Commons Public Domain Dedication waiver (http://creativecommons.org/publicdomain/zero/1.0/) applies to the data made available in this article, unless otherwise stated. 


\section{Background}

Threatened miscarriage is defined as vaginal bleeding with or without abdominal pain and a closed cervical os in early pregnancy. It affects $15-20 \%$ of all pregnancies [1, 2] and is a risk factor for adverse pregnancy outcomes including preeclampsia, pre-term delivery, intrauterine growth restriction, preterm premature rupture of membranes and placental abruption [3]. Amongst women with threatened miscarriage, 15 - 25\% progress to spontaneous miscarriage [4] and they are 2.6 times more likely to miscarry as compared to pregnant women with no bleeding $[5,6]$. Women with threatened miscarriage are often extremely anxious about the pregnancy outcome, and this is not aided by the lack of predictive models that prognosticate and triage such women into the high or low risk of miscarriage [4].

Progesterone is a critical hormone during implantation. It sustains decidualization [7], controls uterine contractility and promotes maternal immune tolerance to the fetal semi-allograft [8]. Lymphocytes, in the presence of progesterone, also release progesterone-induced blocking factor (PIBF). PIBF is a pivotal mediator in progesterone-dependent immunomodulation $[9,10]$ and has a regulatory role in anti-fetal immune responses during pregnancy [11]. One of the earliest studies on progesterone in pregnancy showed an increasing trend of plasma progesterone from conception to delivery [12]. A more recent study by Schock et al further highlighted this increasing trend throughout pregnancy [13]. However, little is known about the distribution of serum progesterone in early pregnancy.

Many studies have shown that low serum progesterone is associated with threatened miscarriage. Our group has validated a single serum progesterone cutoff of $35 \mathrm{nmol} / \mathrm{L}$ taken at presentation with a threatened miscarriage can differentiate women at high or low risk of subsequent miscarriage $[14,15]$. Hence, women with normal pregnancies (low risk) with no bleeding may have a different serum progesterone distribution compared to women with threatened miscarriage. In this study, we aim to establish the distribution of maternal serum progesterone in normal pregnancies and pregnancies complicated by threatened miscarriage from 5 to 13 weeks' gestation.

\section{Methods}

A total of 929 pregnant women, aged 21 years and above, presenting at the KK Women's and Children's Hospital $(\mathrm{KKH})$ antenatal clinics and 24-hour Women's Clinic from January 2013 to December 2016 were recruited. Inclusion criteria were a single intrauterine pregnancy between gestation weeks 5 to 13 (confirmed and dated by ultrasonography), with pregnancy-related per vagina bleeding were recruited in the Threatened Miscarriage [TM] cohort $(n=479)$ while those with no pregnancy-related per vagina bleeding were recruited in the low risk of miscarriage (normal pregnancy [NP]) cohort $(n=450)$. Women with multiple gestations, previous episodes of per vagina bleeding or those treated with progesterone for previous per vagina bleeding in the current pregnancy, or women diagnosed with an inevitable miscarriage, missed miscarriage, blighted ovum or planned termination of pregnancy were excluded.

Maternal blood samples were taken to measure serum progesterone level at presentation as previously described [15]. Blood was collected in plain tubes and centrifuged for $10 \mathrm{~min}$ at $3000 \mathrm{~g}$ within 2 hours of collection. Serum progesterone level was measured in the KKH clinical laboratory using a commercial ARCHITECT progesterone kit (Abbott, Ireland).

Covariates for the analysis were maternal demographics, health, obstetric and lifestyle factors collected by an investigator administered the questionnaire in either English or Chinese (Table 1).

\section{Outcome measures and follow-up}

The primary outcome measured was a spontaneous miscarriage, defined by self-reported uterine evacuation after

Table 1 Serum progesterone and maternal characteristics at baseline, for low risk and high risk women with threatened miscarriage

\begin{tabular}{llll}
\hline Variable & Normal Pregnancy [NP] Cohort $(n=450)$ & Threatened Miscarriage [TM] Cohort $(n=479)$ & $p$-value \\
\hline Demographics & & & $30.6(4.5)$ \\
Maternal Age (yr) & $30.9(4.0)^{\mathrm{a}}$ & $7.3(1.4)$ & $<.058$ \\
Gestational Age (wk) & $8.4(2.1)$ & $23.1(4.6)$ & $<.0001$ \\
Body mass index & $22.9(4.2)$ & 23.0 & 0.459 \\
Previous Miscarriage (\%) & 18.7 & 0.23 & 0.107 \\
Diabetes mellitus (\%) & 0.21 & 4.59 & 1.000 \\
Smoking (\%) & 2.93 & & 0.322 \\
Outcome measures & & $53.6(25.2)$ & $<0.0001$ \\
Serum Progesterone (nmol/L) & $71.8(27.2)$ & 21.5 & $<0.0001$ \\
Miscarriage rate (\%) & 5.4 & &
\end{tabular}


an inevitable or incomplete miscarriage, or complete miscarriage with an empty uterus, by the $16^{\text {th }}$ week of gestation. All participants were contacted at the $16^{\text {th }}$ week of pregnancy to verify pregnancy status.

\section{Statistical Methods}

Baseline maternal demographics and pregnancy characteristics were statistically compared between two study cohorts: (i) patients with no pregnancy-related per vagina bleeding $[\mathrm{NP}]$ and (ii) patients with pregnancy-related per vagina bleeding [TM]. The 2-sample t-test was used to compare continuous baseline variables and Fisher's exact test to compare categorical variables.

Quantile regression was used to characterize serum progesterone levels in the NP and TM cohorts by estimating the 10th, 50th and 90th percentiles from 5 to 13 weeks gestation. Pregnancy outcome was determined at 16 weeks of gestation. Subgroup analysis was carried out within the TM cohort to compare progesterone levels of women who experienced spontaneous miscarriage [TMM] with those who had ongoing pregnancies at 16 weeks of gestation [TMO]. The numbers of patients that presented in each gestation week in the different groups (NP, TM, TMM and TMO) were summarized in Additional file 1: Table S1 and Additional file 2: Figure S1).

This study is funded by the Ministry of Health Industry Alignment Fund Category 1 research fund.

\section{Results}

Miscarriage rates were significantly lower in the normal pregnancy (low risk) [NP] cohort (5.4\%) compared to those who presented with a threatened miscarriage (21.5\%) $(P<0.0001)$. Mean serum progesterone was significantly higher in the NP cohort $(71.8 \pm 27.2 \mathrm{nmol} /$ $\mathrm{L})$ compared to those in the threatened miscarriage [TM] cohort $(53.6 \pm 25.2 \mathrm{nmol} / \mathrm{L})(P<0.0001)$. Women in the NP cohort tend to present later for their booking visit $(8.4 \pm 2.1$ weeks vs $7.3 \pm 1.4$ weeks $)(P<0.0001)$. There were no differences in maternal age, body mass index (BMI), history of previous miscarriages and smoking, or having comorbidities such as diabetes mellitus (Table 1).

Serum progesterone concentration demonstrated a linearly increasing trend from $57.5 \mathrm{nmol} / \mathrm{L}$ to 80.8 $\mathrm{nmol} / \mathrm{L}$ from 5 to 13 weeks gestation in the NP cohort, with a median trend gradient of $\mathrm{b}_{\mathrm{NP}}=2.91(p=$ 0.0020) (Fig. 1, Additional file 1: Table S1A and Additional file 2: Figure S1A). In the TM cohort, serum progesterone concentration increased from $41.7 \mathrm{nmol} /$ $\mathrm{L}$ to $78.1 \mathrm{nmol} / \mathrm{L}$ from 5 to 13 weeks gestation, with a trend gradient of $\mathrm{b}_{\mathrm{TM}}=4.55(p<0.0001)$ (Fig. 1, Additional file 1: Table S1B and Additional file 2: Figure S1B). Median progesterone levels were uniformly lower in the TM cohort by approximately $10 \mathrm{nmol} / \mathrm{L}$,

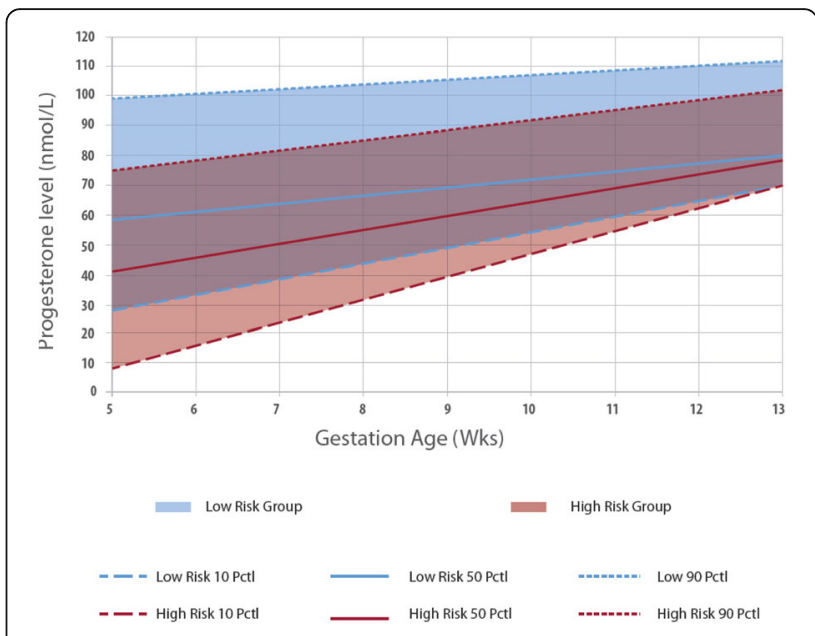

Fig. 1 Distribution of serum progesterone across gestation weeks 5-13 amongst women with normal pregnancy [NP] vs threatened miscarriage [TM]

converging towards the end of the first trimester with similar values at 13 weeks gestation (Fig. 1).

In the subgroup analysis, women in the TM cohort were divided into those with ongoing pregnancies at 16 weeks gestation [TMO] compared to those who experienced spontaneous miscarriage before or at 16 weeks gestation [TMM]. Serum progesterone levels in women with ongoing pregnancy at 16 weeks gestation demonstrated a linearly increasing trend from $47.4 \mathrm{nmol} / \mathrm{L}$ to $75.0 \mathrm{nmol} / \mathrm{L}$ from 5 to 13 weeks gestation, with a trend gradient of $\mathrm{b}_{\mathrm{TMO}}=3.45(p<0.0001)$ (Fig. 2 , Additional file 1: Table S1C and Additional file 2: Figure S1C). Comparatively, there was a non-significant and marginal increase in serum progesterone from $19.0 \mathrm{nmol} / \mathrm{L}$ to $30.3 \mathrm{nmol} / \mathrm{L}$ from 5 to 13 weeks gestation in women who eventually experienced spontaneous miscarriage before or at 16 weeks gestation, with a trend gradient

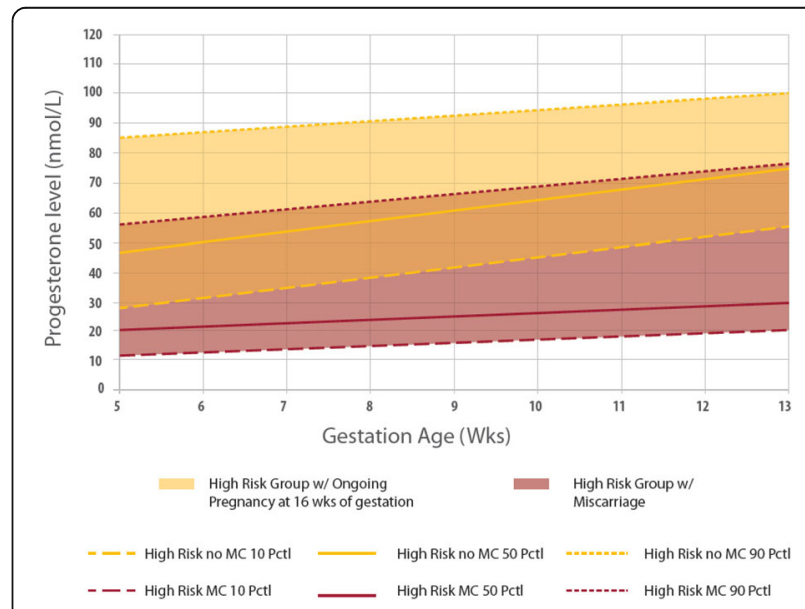

Fig. 2 Threatened miscarriage [TM] subgroup analysis of women with ongoing pregnancy vs spontaneous miscarriage 
of $\mathrm{b}_{\mathrm{TMM}}=1.41(p=0.710)$ (Fig. 2, Additional file 1: Table S1D and Additional file 2: Figure S1D) .

\section{Discussion \\ Main Findings}

Miscarriage rates were significantly lower in the normal pregnancy (low risk) [NP] cohort (5.4\%) compared to those in the threatened miscarriage [TM] cohort. Mean serum progesterone was significantly higher in the NP cohort compared to those in the TM cohort. Serum progesterone increased linearly with gestational age from 5 to 13 weeks in women with normal pregnancies. Women with spontaneous miscarriage showed a marginal and non-significant increase in serum progesterone.

\section{Strengths and Limitations}

This is one of the first prospective cohort studies describing the distribution of serum progesterone in normal pregnancies (low risk) compared to pregnancies that is complicated by threatened miscarriage. There are several limitations of this study. Specifically, the mean gestation at presentation for women in the normal pregnancy cohort is 8.4, while that for women in the threatened miscarriage cohort is 7.3. Women with low risk pregnancies tend to present later, whereas those with bleeding in early pregnancy will seek medical attention promptly. This may be a potential confounder accounting for the higher mean serum progesterone in the normal pregnancy cohort. In addition, the distribution of serum progesterone across gestations is not taken from the same patient, so it may be affected by inherent biological variation amongst patients. Further studies need to be conducted to evaluate the underlying pathophysiology of low progesterone and miscarriage, and examine the role of progestogens in the management of women with threatened miscarriage.

\section{Interpretation}

Many studies have shown that low serum progesterone is associated with poor pregnancy outcomes [16, 17], and our results lend further weight to the pivotal role of progesterone in early pregnancy. In the NP cohort, serum progesterone increased linearly with gestation age from 5 to 13 weeks, with a similar trend observed in TM cohort who had an ongoing pregnancy at 16 weeks gestation.

Progesterone is secreted by the corpus luteum, which only lasts for 14 days if a pregnancy did not occur. In early pregnancy, beta human chorionic gonadotropin ( $\beta$ hCG) secreted by syncytiotrophoblasts maintains the corpus luteum, which allows it to continue secreting progesterone until the placenta takes over its function at 7 to 9 weeks of gestation. Progesterone causes secretory changes in the endometrium of the uterus and is essential for successful implantation of the embryo [18]. Following implantation, elevated levels of circulating progesterone secreted by the placenta acting through progesterone receptors maintain uterine quiescence [19] and stimulate morphological changes to the cervix and other tissues that help to maintain pregnancy [20].

Luteal phase deficiency (LPD) is a condition of insufficient progesterone to maintain a normal secretory endometrium and allow for normal embryo implantation and growth [21]. This is one of many etiologies associated with early pregnancy loss [22]. Two mechanisms have been proposed that results in LPD. The first and likely more common cause relates to the impaired function of the corpus luteum resulting in insufficient progesterone and estradiol secretion [23]. The impaired function can be the result of improper development of the dominant follicle destined to become the corpus luteum or aberrant stimulation of a normally developed follicle, leading to deficiencies in progesterone production. The second mechanism suggests an inability of the endometrium to mount a proper response to appropriate estradiol and progesterone exposure [24].

Apart from LPD, there are other proposed causes of spontaneous miscarriage. More than half of clinically recognized pregnancy loss have been attributed to chromosomal abnormalities [25, 26]. Chromosomal abnormalities could be associated with changes in progesterone levels [27]. Progesterone was shown to be lower in pregnancies with trisomy 13 and trisomy 18 [28]. Other causes of spontaneous miscarriage include maternal factors such as infections and maternal disease states [29].

In women with threatened miscarriage, serum progesterone concentration also increased linearly with gestation, but exhibited a downward displacement of the graph with lower median progesterone levels at every gestation week compared to the low risk group, converging towards the end of the first trimester with similar values at 13 weeks gestation. In women with ongoing pregnancies, vaginal bleeding may be due to disruption of decidual vessels at the maternal-fetal interface [30].

In the subgroup analysis of women with threatened miscarriage, those who experienced a spontaneous miscarriage at or before 16 weeks gestation have a lower serum progesterone level. Many prior studies have shown that the mean serum progesterone level in non-viable gestations are low, ranging between 6.8 $12 \mathrm{ng} / \mathrm{ml}(21.6-38.2 \mathrm{nmol} / \mathrm{L})$ [31-33], but very few have described the distribution of progesterone in early pregnancy. Interestingly, we found that in women with spontaneous miscarriage at or before 16 weeks gestation, there was only a marginal increase in serum progesterone across gestations, with much lower serum progesterone levels between $20 \mathrm{nmol} / \mathrm{L}$ 
to $30 \mathrm{nmol} / \mathrm{L}$. Unlike normal pregnancies, serum progesterone did not increase significantly regardless of gestation in women with spontaneous miscarriage.

\section{Conclusion}

This study highlights the pivotal role of progesterone in supporting an early pregnancy, where lower serum progesterone is associated with threatened miscarriage and a subsequent complete miscarriage at 16 weeks gestation. This may serve as a platform for the development of reference ranges for women who present with low risk pregnancies or threatened miscarriage to predict the risk of subsequent spontaneous miscarriages based on their progesterone levels.

\section{Additional files}

Additional file 1: Table S1A. Distribution of serum progesterone across gestation weeks 5 - 13 amongst women with low risk pregnancy [NP]. Table S1B. Distribution of serum progesterone across gestation weeks 5 - 13 amongst women with threatened miscarriage [TM]. Table S1C. Distribution of serum progesterone across gestation weeks 5 - 13 amongst women who presented with threatened miscarriage and had ongoing pregnancy at 16 weeks [TMO]. Table S1D. Distribution of serum progesterone across gestation weeks 5 - 13 amongst women who presented with threatened miscarriage and had a spontaneous miscarriage at or before 16 weeks [TMM]. (DOCX $20 \mathrm{~kb}$ )

Additional file 2: Figure S1A. Distribution of serum progesterone across gestation weeks $5-13$ amongst women with low risk pregnancy [NP]. Figure S1B. Distribution of serum progesterone across gestation weeks 5 - 13 amongst women with threatened miscarriage [TM]. Figure S1C. Distribution of serum progesterone across gestation weeks $5-13$ amongst women who presented with threatened miscarriage and had ongoing pregnancy at 16 weeks [TMO]. Figure S1D. Distribution of serum progesterone across gestation weeks 5 - 13 amongst women who presented with threatened miscarriage and had a spontaneous miscarriage at or before 16 weeks [TMM]. (ZIP $141 \mathrm{~kb}$ )

\section{Abbreviations}

NP: Normal pregnancy; TM: Threatened miscarriage; TMO: Threatened miscarriage with ongoing pregnancies at 16 weeks gestation;

TMM: Threatened miscarriage with spontaneous miscarriage before or at 16 weeks gestation; KKH: KK Women's and Children's Hospital; BMI: Body mass index; $\beta$ hCG: Beta human chorionic gonadotropin; LPD: Luteal phase deficiency; DM: Diabetes mellitus

\section{Acknowledgements}

The authors will like to thank Ms Trish Koon and Ms Doris Ong for their assistance in patient recruitment. We wish to thank all the families who participated in our research and all the dedicated staff from all participating departments.

\section{Funding}

This study is funded by the Ministry of Health Industry Alignment Fund Category 1 research fund. This facilitated the recruitment of clinical research coordinators who recruited the patients, and also facilitated the analysis and interpretation of data, leading to the writing of the manuscript.

\section{Availability of data and materials}

The datasets generated and/or analyzed during the current study are not publicly available as further research and analysis are being performed on the datasets for future publications but are available from the corresponding author on reasonable request.

\section{Authors' contributions}

CWK developed the research design, analysis strategy, conducted patient recruitment and follow-up and is the first author of the manuscript. JCAJ contributed to the statistical analyses, interpretation of results and presentation and provided editorial guidance. SML developed the research design, analysis strategy, conducted patient recruitment and follow-up. MLC contributed to the analysis and interpretation of results and writing of the manuscript. NST contributed to experimental design for serum progesterone-induced blocking factor quantitation and analysis strategy, interpretation of results and presentation, and provided editorial guidance. TCT contributed to the development of research design, analysis strategy, provided editorial support and is the principal investigator of the Ministry of Health Industry Alignment Fund Category 1 research fund. All authors have reviewed and approved the final version of the paper.

\section{Ethics approval and consent to participate}

The institutional review board at SingHealth (CIRB ref: 2013/320/D) approved the study. All patients have given verbal and written consent to be included in this study.

\section{Consent for publication}

Not applicable.

\section{Competing interests}

The authors declare that they have no competing interests.

\section{Publisher's Note}

Springer Nature remains neutral with regard to jurisdictional claims in published maps and institutional affiliations.

\section{Author details}

'Department of Obstetrics and Gynecology, KK Women's and Children's Hospital, Singapore, 100 Bukit Timah Road, Singapore 229899, Singapore. ${ }^{2}$ Duke-National University of Singapore Graduate Medical School, Singapore, Singapore. ${ }^{3}$ Lee Kong Chian School of Medicine, Nanyang Technological University, Singapore, Singapore. ${ }^{4}$ School of Biological Sciences, Nanyang Technological University, Singapore, Singapore. ${ }^{5}$ Institute of Molecular and Cell Biology, A*STAR, Singapore, Singapore.

Received: 10 February 2018 Accepted: 28 August 2018

Published online: 05 September 2018

\section{References}

1. Ectopic pregnancy and miscarriage (NICE clinical guideline 154). Royal College of Obstetricians and Gynaecologists. 2012. https://www.rcog.org.uk/ en/guidelines-research-services/guidelines/ectopic-pregnancy-andmiscarriage-nice-clinical-guideline-154/. Accessed 26 Feb 2017.

2. Jouppila P. Clinical consequences after ultrasonic diagnosis of intrauterine hematoma in threatened abortion. J Clin Ultrasound. 1985;13(2):107-11.

3. Weiss JL, Malone FD, Vidaver J, Ball RH, Nyberg DA, Comstock CH, et al. Threatened abortion: A risk factor for poor pregnancy outcome, a population-based screening study. Am J Obstet Gynecol. 2004;190:745-50.

4. Basama FMS, Crosfill F. The outcome of pregnancies in 182 women with threatened miscarriage. Arch Gynecol Obstet. 2004;270:86-90

5. Sotiriadis A, Papatheodorou S, Makrydimas G. Threatened miscarriage: evaluation and management. BMJ. 2004;329:152-5.

6. Makrydimas G, Sebire NJ, Lolis D, Vlassis N, Nicolaides KH. Fetal loss following ultrasound diagnosis of a live fetus at 6-10 weeks of gestation. Ultrasound Obstet Gynecol. 2003;22:368-72.

7. Hızlı D, Köşüş N, Köşüş A, Kasap B, Kafali H, Turhan NÖ. First-trimester reference ranges for decidual thickness and its relation to progesterone levels. J Perinat Med. 2012;40(5):521-5.

8. Arck P, Hansen PJ, Jericevic BM, Piccinni MP, Szekeres-Bartho J. Progesterone during pregnancy: Endocrine-immune cross talk in Mammalian Species and the role of stress. Am J Reprod Immunol. 2007;58: 268-79.

9. Szekeres-Bartho J. Progesterone-mediated immunomodulation in pregnancy: its relevance to leukocyte immunotherapy of recurrent miscarriage. Immunotherapy. 2009;1:873-82. https://doi.org/10.2217/imt.09.54. 
10. Szekeres-Bartho J, Barakonyi A, Polgar B, Par G, Faust Z, Palkovics T, et al. The role of gamma/delta $T$ cells in progesterone-mediated immunomodulation during pregnancy: a review. Am J Reprod Immunol. 1999;42:44-8.

11. Kelemen K, Bognar I, Paal M, Szekeres-Bartho J. A progesterone-induced protein increases the synthesis of asymmetric antibodies. Cell Immunol. 1996;167:129-34. https://doi.org/10.1006/cimm.1996.0016.

12. Van Der Molen HJ. Determination of plasma progesterone during pregnancy. Clin Chim Acta. 1963;8(6):943-53.

13. Schock H, Zeleniuch-Jacquotte A, Lundin E, Grankvist K, Lakso H-Å, Idahl A et al. Hormone concentrations throughout uncomplicated pregnancies: a longitudinal study. BMC Pregnancy Childbirth. 2016;16:1-11. https://doi.org/ 10.1186/s12884-016-0937-5.

14. Ku CW, Allen JC Jr, Malhota R, Chong HC, Tan NS, Østbye T, Lek SM, Liw DTT. How can we better predict the risk of spontaneous miscarriage among women experiencing threatened miscarriage? Gynecol Endocrinol. 2015; 31(8):647-51.

15. Lek SM, Ku CW, Allen JC Jr, Malhotra R, Tan NS, Østbye TT. Validation of serum progesterone $<35 \mathrm{nmol} / \mathrm{L}$ as a predictor of miscarriage among women with threatened miscarriage. BMC Pregnancy Childbirth. 2017;17:78.

16. Osmanağaoğlu MA, Erdoğan I, Eminağaoğlu S, Karahan SC, Ozgün S, BH CG. The diagnostic value of beta-human chorionic gonadotropin, progesterone, CA125 in the prediction of abortions. J Obs Gynaecol. 2010;30:288-93.

17. Al Sebai MAH, Kingsland CR, Diver M, Hipkin L, McFadyen IR. The role of a single progesterone measurement in the diagnosis of early pregnancy failure and the prognosis of fetal viability. BJOG An Int J Obstet Gynaecol. 1995;102:364-9.

18. Czyzyk A, Podfigurna A, Genazzani AR, Meczekalski B. The role of progesterone therapy in early pregnancy: from physiological role to therapeutic utility. Gynecol Endocrinol. 2017;33(6):421-4.

19. Mendelson CR. Minireview: fetal-maternal hormonal signaling in pregnancy and labor. Mol Endocrinol. 2009;23:947-54. https://doi.org/10. 1210/me.2009-0016.

20. Byrns MC. Regulation of progesterone signaling during pregnancy: Implications for the use of progestins for the prevention of preterm birth. J Steroid Biochem Mol Biol. 2014;139:173-81.

21. Mesen TB, Young SL. Progesterone and the Luteal Phase: A Requisite to Reproduction. Obstet Gynecol Clin North Am. 2015;42:135-51.

22. Schliep KC, Mumford SL, Hammoud AO, Stanford JB, Kissell KA, Sjaarda LA, et al. Luteal phase deficiency in regularly menstruating women: Prevalence and overlap in identification based on clinical and biochemical diagnostic criteria. J Clin Endocrinol Metab. 2014;99(6):E1007-14.

23. Boutzios G, Karalaki M, Zapanti E. Common pathophysiological mechanisms involved in luteal phase deficiency and polycystic ovary syndrome. Impact on fertility. Endocrine. 2013;43:314-7.

24. Usadi RS, Groll JM, Lessey BA, Lininger RA, Zaino RJ, Fritz MA, et al. Endometrial development and function in experimentally induced luteal phase deficiency. J Clin Endocrinol Metab. 2008;93:4058-64.

25. Enkin M, Keirse M, Neilson J, Crowther C, Duley L, Hodnett E, Hofmeyr J, editors. Guide to Effective Care in Pregnancy and Childbirth. Oxford: Oxford University Press Print; 2000-06.

26. Burgoyne PS, Holland K, Stephens R. Incidence of numerical chromosome anomalies in human pregnancy estimation from induced and spontaneous abortion data. Hum Reprod. 1991;6:555-65. https://www.ncbi.nlm.nih.gov/ pubmed/?term=Burgoyne+PS\%2C+Holland+K\%2C+Stephens+R.+Incidence +of+numerical+chromosome+anomalies+in+human+pregnancy +estimation+from+induced+and+spontaneous+abortion+data.

27. Gillen-Goldstein J, Roque H, Young BK. Steroidogenesis patterns in common trisomies. J Perinat Med. 2002;30:132-6.

28. Kratzer PG, Golbus MS, Monroe SE, Finkelstein DE, Taylor RN. First trimester aneuploidy screening using serum human chorionic gonadotropin (hCG), free ahCG, and progesterone. Prenat Diagn. 1991;11:751-63.

29. Szabó I, Szilágyi A. Management of threatened abortion. Early Pregnancy. 1996;2:233-40. http://www.ncbi.nlm.nih.gov/pubmed/9363221

30. Errol R Norwitz, Joong Shin Park, Charles J Lockwood, Vanessa A Barss Overview of the etiology and evaluation of vaginal bleeding in pregnant women. UpToDate. 2016. https://www-uptodate-com.ezlibproxy1.ntu.edu. sg/contents/overview-of-the-etiology-and-evaluation-of-vaginal-bleeding-inpregnant-women?source=search_result\&search=threatened miscarriage\&selectedTitle=3 150\&id=overview-of-the-etiology-andevaluation-of-vag.
31. Daily CA, Laurent SL, Nunley WC Jr. The prognostic value of serum progesterone and quantitative beta-human chorionic gonadotropin in early human pregnancy. Am J Obstet Gynecol. 1994;171:380-4. http://www.ncbi. $\mathrm{nlm}$.nih.gov/entrez/query.fcgi?cmd=Retrieve\&db=PubMed\&dopt= Citation\&list_uids $=7520212$

32. Rabiee S, Hemmati MFN. Comparison of serum progesterone in non-viable pregnancy and ectopic pregnancy. Acta Med Iran. 2013;51:693-6.

33. Arck PC, Rücke M, Rose M, Szekeres-Bartho J, Douglas AJ, Pritsch $M$, et al. Early risk factors for miscarriage: a prospective cohort study in pregnant women. Reprod Biomed Online. 2008;17:101-13. https://doi.org/10.1016/ S1472-6483(10)60300-8.

\section{Ready to submit your research? Choose BMC and benefit from:}

- fast, convenient online submission

- thorough peer review by experienced researchers in your field

- rapid publication on acceptance

- support for research data, including large and complex data types

- gold Open Access which fosters wider collaboration and increased citations

- maximum visibility for your research: over $100 \mathrm{M}$ website views per year

At BMC, research is always in progress.

Learn more biomedcentral.com/submissions 